\title{
A perspective on terra incognita: uncovering the neuroanatomy of the human subcortex
}

\author{
Anneke Alkemade ${ }^{1 \dagger}$, Max C. Keuken ${ }^{1,2 t}$ and Birte U. Forstmann ${ }^{1,2 *}$ \\ ${ }^{1}$ Department of Psychology, Cognitive Science Center Amsterdam, University of Amsterdam, Amsterdam, Netherlands \\ ${ }^{2}$ Department of Neurophysics, Max Planck Institute for Human Cognitive and Brain Sciences, Leipzig, Germany
}

\section{Edited by:}

George Paxinos, University of New

South Wales, Australia

Reviewed by:

Noritaka Ichinohe, National Center of Neurology and Psychiatry, Japan

Ruth Luthi-Carter, University of

Leicester, UK

*Correspondence:

Birte U. Forstmann, Department of Psychology, Cognitive Science

Center Amsterdam, University of

Amsterdam, Nieuwe Achtergracht

130, 1018VZ Amsterdam,

Netherlands

e-mail:buforstmann@gmail.com

${ }^{+}$These authors have contributed

equally to this work.
Recent exciting advancements in the field of in vivo neuroimaging allow for visualization of the living human brain with unprecedented anatomical detail. Large consortium studies will provide us with novel insights in the function and connectivity of the human brain. However, it is unlikely that the spatial resolution obtained using in vivo imaging will, in the near future, approximate the level of detail obtained in post-mortem anatomical studies. Initiatives such as the recently published Big Brain project (Amunts et al., 2013) herald a novel approach in post-mortem brain research. We feel that linking data from histological observations with in vivo imaging studies will greatly advance our understanding of the functional neuroanatomy of the human brain.

Keywords: Human brain atlas, subcortical, ex vivo, in vivo
It is likely that in vivo imaging techniques will develop further during the next decade, allowing visualization of the human brain with unprecedented detail. Although the technical developments in in vivo brain imaging are exciting, the anatomical detail that is obtained remains limited. Increasing field strength of magnetic resonance imaging (MRI) scanners will improve the spatial resolution even further. However, it is unlikely that in vivo MRI will yield a level of detail comparable to histological approaches for studying the brain. At present a standard anatomical scan, obtained using a 3 Tesla MRI system, will take about $6 \mathrm{~min}$ and has an isotropic resolution of $1 \mathrm{~mm}$. Such resolution does not allow the identification of individual neurons or glia, which can be achieved using histological techniques (Duyn, 2012). We argue that combining of post-mortem research with modern imaging techniques is needed to advance the research field of neuroscience.

Using state-of-the-art ultra-high resolution MRI, we can now visualize a number of small subcortical areas such as the subthalamic nucleus (Schafer et al., 2012). Although this is impressive, comparing these scans to images of histological staining of the same area puts in vivo MRI images into perspective. The detail obtained using ultra-high resolution MRI does not begin to compare to that of histology. Histological techniques have their limitations as well, and are typically used to obtain information about tissues in a 2D plane. Only small parts of the brain are usually studied for practical reasons. It seems only logical to combine the best of both worlds, but this rarely happens.

An unusually detailed, and impressive study was recently published by Amunts et al. (2013). In this study, an entire human brain obtained at autopsy was fixed, and cut in a coronal plane. Cytoarchitectonic structures were visualized using silver staining, microscope sections were photographed, and then with modern computational techniques further processed ultimately resulting in a $3 \mathrm{D}$ reconstruction with unprecedented detail, known as the Big Brain. This work is impressive not only in view of the spatial resolution it provides, but also in its laborious nature. More concretely, the resolution was $20 \mu \mathrm{m}$ isotropic, required $1000 \mathrm{~h}$ of work, and resulted in $1 \mathrm{~Tb}$ of raw data. One can easily calculate the needed amount of work for the generalization of these results to the population. In this perspective article, we argue that such efforts are urgently needed to help define terra incognita, i.e., the human subcortex, in modern maps of the brain.

The lack of MRI research on small brain structures, especially subcortical structures, is reflected by their absence from brain atlases currently available for MRI research (Evans et al., 2012). We have illustrated this (Figure 1) by comparing an inventory of the subcortical gray matter structures depicted in standard MRI-atlases with the structures defined in the Federative Community on Anatomical Terminology (FCAT, 1998); a standardized nomenclature on human brain structures. The discrepancy should serve as a wakeup call for neuroscientists. We calculated that approximately seven percent of the structures mentioned in the FCAT are depicted in available standard MRIatlases. This implies that these structures cannot be studied using automated analytical protocols available for MRI, and indicates the necessity of trained anatomists for the study of subcortical brain areas.

Neuroscientists around the world collaborate in order to fill our neuroanatomical knowledge-gap. The human connectome project is a 5 years collaborative project between 36 scientists from 11 different institutions, aimed to clarify the connectivity within the human brain in a cohort of 1200 healthy adults, using 3 and 7 Tesla MRI, diffusion tensor imaging, resting state fMRI, task fMRI, MEG, EEG, behavioral testing, DNA-analyses and network modeling (http://www.humanconnectomeproject.org). 


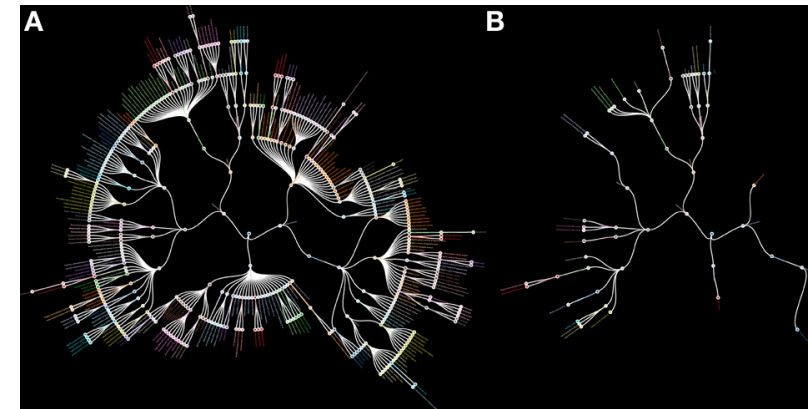

FIGURE 1 | Radial hierarchical tree of the human subcortex. (A) A radia hierarchical tree illustrating the 455 subcortical structures as defined by the Federative Community on Anatomical Terminology (FCAT). The outer edges of the hierarchical tree display the individual subcortical gray matter nuclei. (B) A radial hierarchical tree illustrating seven percent of all subcortical structures as defined by the FCAT that are implemented as standard atlas maps in the three major MRI analysis software packages: FSL, SPM, and Freesurfer.

These studies are aimed to provide insight in the connectivity between gray areas within the normal human brain. The human connectome project will undoubtedly provide us with exciting new insights in the functional connections within the human brain. Such an ambitious approach would entail manual segmentation of all subcortical gray matter structures of the human brain. Our and other research groups are taking humble steps to start the mapping of subcortical structures in in vivo MRI scans (Cho et al., 2010; Kwon et al., 2012; Lenglet et al., 2012; Keuken et al., 2013). Our recent efforts indicate that mapping a single structure in 30 MRI scans takes approximately 4 months of work by two researchers. If we extrapolate these experiences to the human connectome project, it would take approximately 12,000 years of work to segment the 455 subcortical gray matter structures listed in the FCAT.

We live in an exciting era, and we feel that the continuing developments in functional and structural MRI research are invaluable for increasing understanding of the human brain. However, technical advancements in in vivo imaging do not abolish the need for classical, laborious anatomical studies. We believe that linking data obtained from the Big Brain project, the human connectome, in combination with other initiatives, such as the Allen Brain Atlas (www.brain-map.org), Blue Brain (bluebrain.epfl.ch), Human Brain project (www. humanbrainproject.eu), and the BRAIN initiative (www.nih.gov/ science/brain) will contribute to improve our understanding of the human subcortex. Further developments in ultra-high field MRI stand at the brink of our journey of accurate and detailed mapping of the human brain. Obtaining detailed neuroanatomical maps of the human subcortex and registration of these maps into standard space will further facilitate and advance fMRI research.

\section{REFERENCES}

Amunts, K., Lepage, C., Borgeat, L., Mohlberg, H., Dickscheid, T., Rousseau, M. E. et al. (2013). BigBrain: an ultrahigh-resolution 3D human brain model. Science 340, 1472-1475. doi: 10.1126/science.1235381

Cho, Z. H., Min, H. K., Oh, S. H., Han, J. Y., Park, C. W., Chi, J. G., et al. (2010). Direct visualization of deep brain stimulation targets in Parkinson disease with the use of 7-tesla magnetic resonance imaging. J. Neurosurgery 113, 1-9. doi: 10.3171/2010.3.JNS091385

Duyn, J. H. (2012). The future of ultra-high field MRI and fMRI for study of the human brain. Neuroimage 62, 1241-1248. doi: 10.1016/j.neuroimage.2011. 10.065

Evans, A. C., Janke, A. L., Collins, D. L., and Baillet, S. (2012). Brain templates and atlases. Neuroimage 62, 911-922. doi: 10.1016/j.neuroimage.2012.01.024

Federative Committee on Anatomical Terminology. (ed.). (1998). Terminologia Anatomica. New York, NY: Thieme Stuttgart.

Keuken, M. C., Bazin, P. L., Schafer, A., Neumann, J., Turner, R., and Forstmann, B. U. (2013). Ultra-high 7T MRI of structural age-related changes of the subthalamic nucleus. J. Neurosci. 33, 4896-4900. doi: 10.1523/JNEUROSCI.324112.2013

Kwon, D.-H., Kim, J.-M., Oh, S.-H., Jeong, H.-J., Park, S.-Y., Oh, E.-S., et al. (2012). Seven-tesla magnetic resonance images of the substantia nigra in Parkinson disease. Ann. Neurol. 71, 267-277. doi: 10.1002/ana.22592

Lenglet, C., Abosch, A., Yacoub, E., De Martino, F., Sapiro, G., and Harel, N. (2012). Comprehensive in vivo mapping of the human basal ganglia and thalamic connectome in individuals using 7T MRI. PLoS ONE 7:e29153. doi: 10.1371/journal.pone.0029153

Schafer, A., Forstmann, B. U., Neumann, J., Wharton, S., Mietke, A., Bowtell, R., et al. (2012). Direct visualization of the subthalamic nucleus and its iron distribution using high-resolution susceptibility mapping. Hum. Brain Mapp. 33, 2831-2842. doi: 10.1002/hbm.21404

Conflict of Interest Statement: The authors declare that the research was conducted in the absence of any commercial or financial relationships that could be construed as a potential conflict of interest.

Received: 16 September 2013; paper pending published: 28 October 2013; accepted: 18 November 2013; published online: 03 December 2013.

Citation: Alkemade A, Keuken MC and Forstmann BU (2013) A perspective on terra incognita: uncovering the neuroanatomy of the human subcortex. Front. Neuroanat. 7:40. doi: 10.3389/fnana.2013.00040

This article was submitted to the journal Frontiers in Neuroanatomy.

Copyright (c) 2013 Alkemade, Keuken and Forstmann. This is an open-access article distributed under the terms of the Creative Commons Attribution License (CC BY). The use, distribution or reproduction in other forums is permitted, provided the original author(s) or licensor are credited and that the original publication in this journal is cited, in accordance with accepted academic practice. No use, distribution or reproduction is permitted which does not comply with these terms. 